Correspondence

Mark C. Herzberg

mcherzb@umn.edu

Received 12 March 2009

Revised 7 July 2009

Accepted 10 July 2009

\section{Cleavage of protease-activated receptors on an immortalized oral epithelial cell line by Porphyromonas gingivalis gingipains}

\author{
Rodrigo A. Giacaman, ${ }^{1,2} \dagger$ Anil C. Asrani, ${ }^{1,2}$ Karen F. Ross ${ }^{1,2}$ \\ and Mark C. Herzberg ${ }^{1,2}$ \\ ${ }^{1}$ Department of Diagnostic and Biological Sciences, School of Dentistry, University of Minnesota, \\ Minneapolis, MN 55455, USA \\ ${ }^{2}$ The Mucosal and Vaccine Research Center, Minneapolis VA Medical Center, Minneapolis, MN \\ 55417, USA
}

\begin{abstract}
Porphyromonas gingivalis activates protease-activated receptors (PARs) on oral keratinocytes, resulting in downstream signalling for an innate immune response. Activation depends on $P$. gingivalis gingipains, but could be confounded by lipopolysaccharide signalling through Toll-like receptors. We therefore hypothesized that $P$. gingivalis cleaves oral keratinocyte PARs in an Arg(Rgp) or Lys- (Kgp) gingipain-specific manner to upregulate pro-inflammatory cytokines. Immortalized human oral keratinocytes (TERT-2) were incubated with wild-type $P$. gingivalis (ATCC 33277) or strains from a panel of isogenic gingipain deletion mutants: Kgp-deficient (KDP 129); Rgp-deficient (KDP 133); or Kgp- and Rgp-deficient (KDP 136). After incubation with $P$. gingivalis, keratinocytes were probed with specific antibodies against the N-terminus of PAR-1 and PAR-2. Using flow cytometry and immunofluorescence, receptor cleavage was marked by loss of specific antibody binding to the respective PARs. TERT-2 cells constitutively expressed high levels of PAR-1 and PAR-2, and lower levels of PAR-3. P. gingivalis ATCC 33277 cleaved PAR-1 and PAR-2 in a dose-dependent manner, while the receptors were unaffected by the protease-negative double mutant (KDP 136) at all m.o.i. tested. The single Kgp-negative mutant preferentially cleaved PAR-1, whereas the Rgp-negative mutant cleaved PAR-2. Wild-type or Kgp-negative mutant cleavage of PAR- 1 upregulated expression of IL- $1 \alpha$, IL- $1 \beta$, IL- 6 and TNF- $\alpha$; the Rgp-negative mutant did not modulate these cytokines. Selective cleavage of PAR-1 on oral epithelial cells by $P$. gingivalis Rgp therefore upregulates expression of pro-inflammatory cytokines.
\end{abstract}

\section{INTRODUCTION}

Porphyromonas gingivalis is a Gram-negative anaerobe present in subgingival plaque and widely associated with periodontitis in adults (Chen et al., 2001; Nonnenmacher et al., 2004, 2005). Periodontal diseases and inflammation are associated with specific $P$. gingivalis virulence factors (Tatakis \& Kumar, 2005), including fimbriae (Miura et al., 2005; Weinberg et al., 1997), lipopolysaccharides (LPS) (Wang et al., 2002) and gingipains (Kadowaki et al., 1994, 2003; Pathirana et al., 2007a; Potempa et al., 2000; Yoneda et al., 1990). Gingipains are cysteine proteases produced by P. gingivalis (Kadowaki et al., 1994; Potempa et al., 2000). Two types of gingipains have been described: arginine-

tPresent address: Departamento de Rehabilitación Buco-Maxilofacial, Universidad de Talca, Talca, Chile.

Abbreviations: Kgp, lysine-specific gingipain(s); PAR, protease-activated receptor; Rgp, arginine-specific gingipain(s); TLR, Toll-like receptor. specific (Arg-gingipains or Rgp) and lysine-specific (Lys-gingipains or Kgp). Rgp can be found as three variants: a $50 \mathrm{kDa} \mathrm{RgpB}$, a $50 \mathrm{kDa} \mathrm{RgpA}_{\text {cat }}$ and a $95 \mathrm{kDa}$ HRgpA (Potempa et al., 2000). In a mouse model, Kgp and RgpB, but not RgpA, contribute to periodontal disease (Pathirana et al., 2007a), findings that were confirmed by the use of novel gingipain inhibitors (Kadowaki \& Yamamoto, 2003; Kadowaki et al., 2004).

On eukaryotic cell membranes, gingipains cleave the Nterminal domains to activate the protease-activated receptors (PARs), members of the seven-transmembrane superfamily of cell-surface G-protein-coupled receptors (reviewed by Coughlin, 2000; Ossovskaya \& Bunnett, 2004). Four PARs are currently described: PAR-1, PAR-2, PAR-3 and PAR-4. Hence, $P$. gingivalis gingipains cleave and activate PARs on neutrophils (Lourbakos et al., 1998) and platelets (Lourbakos et al., 2001b), and Rgp activation of PAR-2 has been linked to inflammation and induction 
of alveolar bone loss in periodontitis (Holzhausen et al., 2005, 2006). Consistent with these findings, PAR-2deficient mice lose less alveolar bone than wild-type mice after challenge with $P$. gingivalis supernatants, which appears to be caused by Rgp signalling through PAR-2 (Holzhausen et al., 2006). In KB cells (a HeLa-like carcinoma cell line), however, $P$. gingivalis Rgp appears to activate PAR- 1 and PAR-2 and release IL-6, a potent stimulator of osteoclast differentiation and bone resorption (Lourbakos et al., 2001a). Hence, the PAR-gingipain specificity leading to release of pro-inflammatory cytokines is unclear, in part complicated by the presence of $P$. gingivalis LPS with concomitant activation through Tolllike receptors (TLRs) also leading to expression of proinflammatory cytokines (Chen et al., 2008; Diya et al., 2008; Eskan et al., 2008).

In the present study, we determined the $P$. gingivalis gingipain cleavage specificity for PARs on oral keratinocytes. By using an immortalized oral epithelial cell line, TERT-2, we have shown that PAR-1 and PAR-2 are differentially cleaved by $P$. gingivalis Rgp and Kgp to upregulate expression of pro-inflammatory cytokines.

\section{METHODS}

Cell cultures. OKF6/TERT-2 (TERT-2), an immortalized oral epithelial cell line provided by J. Rheinwald (Harvard Medical School, Cambridge, MA) (Dickson et al., 2000), was used to model primary gingival epithelial cells. TERT-2 cells were grown in $5 \% \mathrm{CO}_{2}$ at $37{ }^{\circ} \mathrm{C}$ in keratinocyte serum-free and calcium-free medium (Invitrogen Life Technologies) supplemented with $0.4 \mathrm{mM} \mathrm{CaCl}$, $25 \mu \mathrm{g} \mathrm{ml} l^{-1}$ bovine pituitary extract and $0.2 \mathrm{ng} \mathrm{ml}^{-1}$ epidermal growth factor, as previously reported (Giacaman et al., 2007). Culture medium was changed every 3 days and cells were subcultured when they reached approximately $60 \%$ confluence.

Bacterial strains and culture conditions. $P$. gingivalis strain ATCC 33277 and a panel of isogenic deletion mutants that fail to express Kgp (KDP 129; $\Delta$ kgp) (Okamoto et al., 1998), Rgp (KDP 133; $\Delta r g p A$ $\Delta r g p B$ ) (Nakayama et al., 1995) or both gingipains (KDP 136; $\Delta k g p$ $\Delta \operatorname{rgp} A \Delta r g p B$ ) (Shi et al., 1999) (kindly provided by K. Nakayama, Nagasaki University, Nagasaki, Japan) were used. P. gingivalis strains were grown anaerobically in a Coy chamber $\left(85 \% \mathrm{~N}_{2}, 5 \% \mathrm{CO}_{2}\right.$ and $10 \% \mathrm{H}_{2}$ ) at $37{ }^{\circ} \mathrm{C}$ on Todd-Hewitt agar plates (Difco) or in ToddHewitt broth. Agar and broth were supplemented with $5 \mu \mathrm{g}$ haemin $\mathrm{ml}^{-1}$ and $1 \mu \mathrm{g}$ menadione $\mathrm{ml}^{-1}$ (both Sigma-Aldrich). Agar plates were also supplemented with $5 \%(\mathrm{v} / \mathrm{v})$ defibrinated sheep blood. Bacteria were grown to early stationary-phase $\left(\mathrm{OD}_{620} \sim 0.8-1.0\right)$. Spent bacterial broth with early stationary phase $P$. gingivalis cells containing secreted and cell-wall-associated gingipains (Potempa et al., 2000) was added to keratinocyte cultures at $37^{\circ} \mathrm{C}$ at an m.o.i. (bacteria to keratinocytes) of $10: 1,100: 1$ or $1000: 1$. In preparation, keratinocytes at $60 \%$ confluence were washed with Dulbecco's PBS (DPBS) (Mediatech) and the medium was replaced with pre-warmed serum-free MEM (Mediatech). In the presence of inoculated $P$. gingivalis, TERT-2 cells were incubated at $37{ }^{\circ} \mathrm{C}$ in $5 \% \mathrm{CO}_{2}$ for $1 \mathrm{~h}$. Control TERT-2 cells were inoculated with fresh sterile $P$. gingivalis growth medium and maintained under the same conditions. For some control experiments, TERT-2 cells were incubated for $30 \mathrm{~min}$ in serum-free MEM (Mediatech) at $37{ }^{\circ} \mathrm{C}$ in $5 \% \mathrm{CO}_{2}$ with either $100 \mathrm{nM} \quad \mathrm{N}$-tosyl-L-phenylalanine chloromethyl ketone (TPCK)treated trypsin (Sigma-Aldrich) or $100 \mathrm{nM} \Delta$-thrombin (MP
Biomedicals). After incubation with $P$. gingivalis, sterile control medium, TPCK-trypsin or thrombin, keratinocytes were washed three times with DPBS and processed for the appropriate analysis as below.

Flow cytometry. After incubation with $P$. gingivalis or controls, TERT-2 cells were washed three times with DPBS and detached from the flasks or wells using $0.2 \%(\mathrm{w} / \mathrm{v})$ EDTA and gentle scraping. Since trypsin activates PARs (Lourbakos et al., 2001a), this enzyme was not used to detach the cells. Cells were counted, fixed for 10 min at $4{ }^{\circ} \mathrm{C}$ in $4 \%(\mathrm{w} / \mathrm{v})$ paraformaldehyde and reacted with optimized dilutions of primary antibodies against PAR-1, -2 and -3 . The antibodies used were murine monoclonal antibodies against PAR-1 (ATAP) (Santa Cruz Biotechnology) or PAR-2 (Zymed), or rabbit polyclonal antibody against PAR-3 (Santa Cruz Biotechnology). Primary antibodies and cells were incubated for $30 \mathrm{~min}$ at $4{ }^{\circ} \mathrm{C}$, washed three times with PBS supplemented with $2 \%(\mathrm{v} / \mathrm{v})$ FBS, and washed cells were then incubated in the dark for 30 min with a $1: 100$ dilution of goat anti-murine IgG secondary antibody conjugated with phycoerythrin (Jackson ImmunoResearch Laboratories) to detect PAR-1 or PAR-2, or with goat anti-rabbit IgG secondary antibody conjugated with FITC (Sigma) for PAR-3. Murine monoclonal antibodies IgG1 and IgG2a (BD Biosciences and BD Pharmingen) were used as isotype controls for PAR-1 and PAR-2, respectively. For PAR-3 detection, rabbit polyclonal IgG was used as isotype control. Fluorescent signals were monitored with a FACScan (BD Biosciences) using CellQuest software. Each antibody was analysed and reactivity was confirmed in at least three independent experiments.

Immunofluorescence microscopy. TERT-2 cells $\left(2 \times 10^{5}\right)$ were grown as described above and seeded on coverslips in 12-well plates. Cells $(60 \%$ confluent) were incubated with $P$. gingivalis for $1 \mathrm{~h}$ at various m.o.i. After incubation with $P$. gingivalis, cells were washed three times with DPBS, fixed in $4 \%(\mathrm{w} / \mathrm{v})$ paraformaldehyde, and blocked with $3 \%(\mathrm{w} / \mathrm{v})$ BSA for $30 \mathrm{~min}$ at room temperature to minimize non-specific antibody binding. To detect PARs, TERT-2 cells were incubated with $80 \mu \mathrm{l}$ of a $1: 100$ dilution of murine monoclonal antibody against PAR-1 (ATAP) or PAR-2 (SAM-11) (Santa Cruz Biotechnology) for $1 \mathrm{~h}$ in the dark. Murine IgG1 and IgG2a antibodies were used as isotype controls for PAR-1 and PAR-2, respectively. Cells were washed five times with DPBS and then incubated in the dark for one additional hour with Alexa Fluor 568conjugated goat anti-murine IgG Antibody (Molecular Probes) and 4',6-diamidino-2-phenylindole (DAPI; Molecular Probes) at 1:2000 and $1: 300$ dilutions, respectively. Coverslips were washed five times, mounted on glass slides, and cells were observed using a Nikon Eclipse E800 fluorescence microscope. All experiments were performed in triplicate.

Quantitative PCR. Since incubation with $P$. gingivalis for longer than $6 \mathrm{~h}$ causes cells to detach from the flasks (Nisapakultorn et al., 2001), keratinocytes were incubated in TERT medium with $P$. gingivalis for $3 \mathrm{~h}$, as previously described (Giacaman et al., 2007), washed five times with DPBS and then incubation continued for the times indicated. At times up to $48 \mathrm{~h}$ after initial exposure to $P$. gingivalis, TERT-2 cells were harvested. Total RNA was extracted using an Rneasy Plus Mini kit (Qiagen) according to the manufacturer's instructions, quantified using a 2100 Bioanalyser (Agilent Technologies), and $500 \mathrm{ng}$ was used as template to synthesize cDNA using the Superscript III First Strand Synthesis System for RT-PCR (Invitrogen). The cDNA was then diluted 1:5 with RNase/DNase-free water and $1 \mu \mathrm{l}(5 \mathrm{ng})$ was used as template in the Platinum SYBR Green qPCR SuperMix-UDG with ROX (Invitrogen). Quantitative PCR was performed on each sample in triplicate using an ABI7900 HT (Applied Biosystems). Data were analysed using SDS 2.1 software (Applied Biosystems), normalizing all genes to human $\beta$-actin (SuperArray Bioscience). $\mathrm{RT}^{2}$ qPCR primers (SuperArray Bioscience) were used to detect specific mRNAs for the cytokines IL- $1 \alpha$, IL- $1 \beta$, IL- 6 , TNF- $\alpha$, IL- 8 and IFN- $\gamma$. At each time 
point, expression of the selected mRNAs in cells incubated with $P$. gingivalis was calculated relative to the housekeeping gene $\beta$-actin $\left(\Delta \mathrm{C}_{\mathrm{t}}\right)$ for each sample, and then expressed relative to untreated cells at the same time point using the $2^{-\Delta \Delta C_{\mathrm{t}}}$ method (Livak \& Schmittgen, 2001).

Statistical analyses. Statistical analyses were performed by Student's $t$ test for paired values using GraphPad software (GraphPad Software). Differences were considered significant at $P<0.05$.

\section{RESULTS}

\section{Expression of PAR-1, PAR-2 and PAR-3 on oral epithelial cells}

To learn whether TERT-2 cells constitutively express surface PARs, resting, untreated keratinocytes were analysed by flow cytometry for PAR-1, PAR-2 and PAR-3. TERT-2 cells expressed surface PAR-1, -2 and -3 (Fig. 1). PAR-1 and PAR-2 were more frequently expressed at detectable levels than PAR-3 $(P<0.001)$.

\section{Thrombin and trypsin effects on PAR cleavage}

Since thrombin and trypsin cleave PARs (Hansen et al., 2004), we treated TERT-2 cells to confirm cleavage of PAR1 and PAR-2. Compared to levels on untreated cells, PAR-1 was significantly cleaved ( $60 \%$ reduction) by thrombin (Fig. 2A, E), but PAR-2 was unaffected (Fig. 2B, I). In contrast, trypsin cleaved both PAR-1 (Fig. 2A, F) and PAR2 (Fig. 2B, J) to barely detectable levels. Although PAR-3 was detected at low levels (Fig. 1), thrombin and trypsin apparently did not cleave this receptor (data not shown). For all subsequent experiments, thrombin and trypsin were used as positive controls for PAR-1, and PAR-1 and PAR-2 cleavage, respectively.

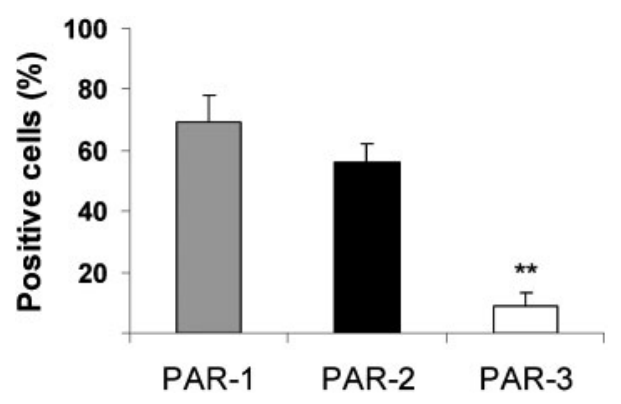

Fig. 1. PAR expression in oral keratinocytes. TERT-2 cells, grown as described in Methods, were analysed by flow cytometry to assess constitutive expression of surface PAR-1, PAR-2 and PAR3. Cells were seeded in flasks and harvested without trypsin as described. Bars represent the mean \pm SEM of five independent experiments. ${ }^{* \star} P<0.001$, relative to PAR- 1 .

\section{P. gingivalis cleavage of PAR-1 and PAR-2 receptors on oral epithelial cells}

TERT-2 cells were incubated for $1 \mathrm{~h}$ with $P$. gingivalis ATCC 33277 in spent medium at various m.o.i. to verify cleavage of PARs. P. gingivalis cleaved PAR-1 (Fig. 3A) and PAR-2 (Fig. 3B) in a dose-dependent manner. PAR-1 (Fig. $3 \mathrm{~A}$ ) was cleaved more effectively than PAR-2 at each m.o.i. tested (Fig. 3B) and PAR-2 cleavage was only detectable at m.o.i. greater than 100:1 (Fig. 3B). At m.o.i. 100:1 and $1000: 1$, P. gingivalis reduced PAR-1 signals to levels comparable to trypsin treatment and similar to untreated cells incubated with the isotype control antibody (Fig. 3A). Cleavage of the PARs by $P$. gingivalis was confirmed by immunofluorescence microscopy (Fig. 3C-L).

\section{Effect of gingipains on PAR cleavage}

To determine whether gingipains produced by intact cells of $P$. gingivalis were responsible for PAR cleavage, keratinocytes were incubated with an isogenic mutant strain, KDP 136, which fails to express either gingipain. Unlike the wild-type, KDP 136 was unable to cleave either PAR-1 or PAR-2 at any m.o.i. tested up to 1000:1 (data not shown). To investigate the role of each $P$. gingivalis cysteine protease on PAR cleavage, TERT-2 cells were incubated with a Lys- (KDP 129) or Arg-gingipain-deletion mutant (KDP 133), or with the wild-type ATCC 33277. P. gingivalis KDP 129 cleaved PAR-1 (Fig. 4A), but not PAR-2 (Fig. 4B), suggesting that cleavage of PAR-1 was Arggingipain specific. When oral keratinocytes were incubated with KDP 133, which does not express the Arg-gingipains (Fig. 4C, D), the percentage of PAR-1 positive cells was only slightly reduced (Fig. 4C) whereas the percentage of PAR-2 positive cells was reduced to levels similar to the wild-type strain (Fig. 4D). This suggested that PAR-2 cleavage was largely Lys-gingipain-dependent. Since PAR-2 was significantly cleaved by the wild-type, cleaved only moderately by the Rgp-deficient mutant and was unaffected by the double mutant, the two proteases are suggested to act synergistically to cleave PAR-2.

\section{Gingipain-mediated regulation of pro-inflammatory cytokines}

To determine which gingipain upregulates expression of pro-inflammatory cytokines, oral keratinocytes were incubated with $P$. gingivalis ATCC 33277 or the gingipain mutant strains. IL- $1 \alpha$, IL- $1 \beta$, IL- 6 , TNF- $\alpha$, IL- 8 and IFN- $\gamma$ expression was analysed by real-time PCR. By $6 \mathrm{~h}, P$. gingivalis induced significant increases in the expression of pro-inflammatory cytokines IL- $1 \alpha$, IL- $1 \beta$, IL- 6 and TNF- $\alpha$ (Fig. 5A-D), but not IL-8 and IFN- $\gamma$ (data not shown), by TERT-2 cells. Furthermore, upregulation of IL- $1 \beta$, IL-6, IL$1 \alpha$ and TNF- $\alpha$ appeared to be gingipain-dependent. While $\Delta \mathrm{Kgp}$ (KDP 129) and the wild-type induced expression of a similar cytokine profile, the $\Delta \operatorname{Rgp}(\mathrm{KDP} 133$ ) and the double mutant KDP 136 were unable to upregulate the 
A

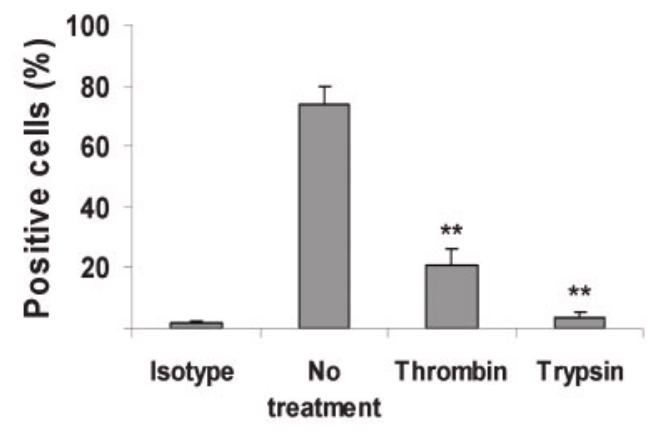

B

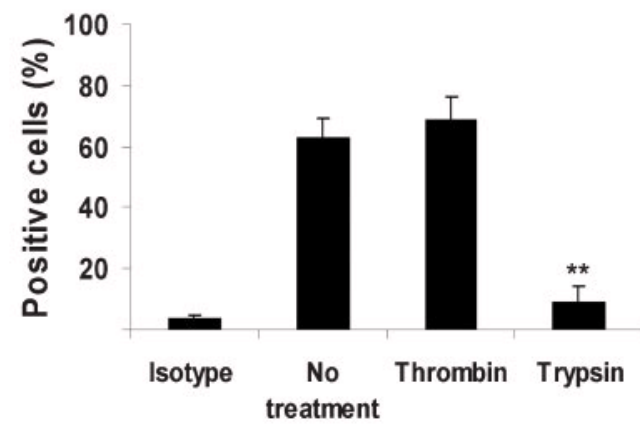

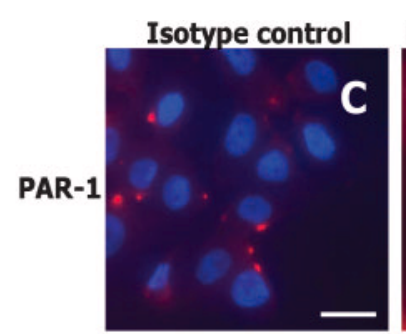

No treatment (medium)
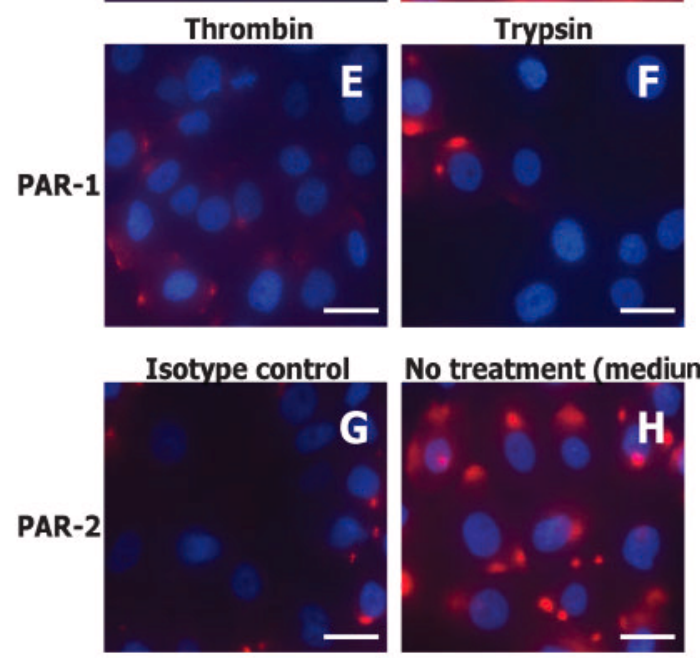

No treatment (medium)

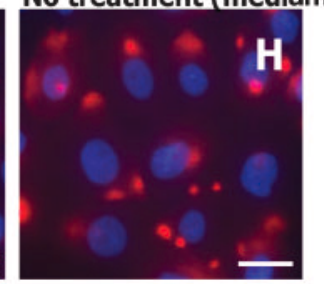

Thrombin

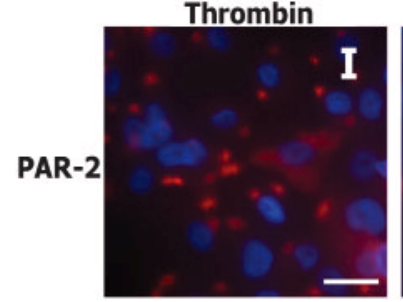

Trypsin

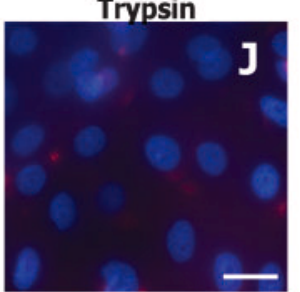

Fig. 2. PAR cleavage by thrombin and trypsin. Oral keratinocytes were incubated with medium, thrombin or trypsin (100 nM) for $30 \mathrm{~min}$ at $37{ }^{\circ} \mathrm{C}$ in $5 \% \mathrm{CO}_{2}$. For flow cytometry (A, B), cells were detached from the vessels using $0.2 \%$ (w/v) EDTA, fixed, blocked, stained with isotype IgG, PAR-1 or PAR-2 antibodies, and analysed to determine protease cleavage of PAR-1 (A) and PAR-2 (B) on the cell surface. For immunofluorescence (C-J), cells were incubated with either fresh medium $(C, D, G, H)$ or proteases $(E, F, I, J)$, fixed, blocked and stained with isotype control $\lg G(C$ and $G$ ) or antibodies against PAR-1 (D-F) or PAR-2 $(\mathrm{H}-\mathrm{J})$, as described. Bars represent the mean \pm SEM of three independent experiments. ${ }^{\star \star} P<0.001$ when compared to 'No treatment'. For both assays, $\lg G 1(A, C)$ and $\lg G 2 a(B, G)$ isotype antibodies were used as negative controls at the same concentration as antibodies used to detect PAR. Scale bars, $8 \mu \mathrm{m}$. Blue, DAPI; red, Alexa Fluor 568-conjugated IgG. Images are representative of two independent experiments.

pro-inflammatory cytokines. These findings suggest that Arg-gingipains selectively cleave PAR-1 to upregulate proinflammatory cytokines in oral keratinocytes.

\section{DISCUSSION}

Oral keratinocytes express pro-inflammatory cytokines in response to indigenous pathogens such as $P$. gingivalis. As we have reported (Giacaman et al., 2007), P. gingivalis Rgp upregulates CCR5 through PAR-1 and -2 signalling. Hence, the PARs enhance the responses of keratinocytes to cytokines. In addition to selective upregulation of chemo- kine receptors, we now provide strong evidence that PAR signalling also increases the expression of pro-inflammatory cytokines, exclusive of LPS-mediated responses through TLRs (reviewed by Potempa \& Pike, 2009).

Virulence of $P$. gingivalis against oral epithelial cells has often been characterized in KB cells (Eick et al., 2006; Madianos et al., 1996; Pathirana et al., 2007b). Although $\mathrm{KB}$ cells were originally derived from an oral epidermal carcinoma, further analysis demonstrated HeLa markers in the chromosomes. KB cells are now considered HeLa contaminants (American Type Culture Collection, ATCC). In our studies we used an immortalized oral keratinocyte 

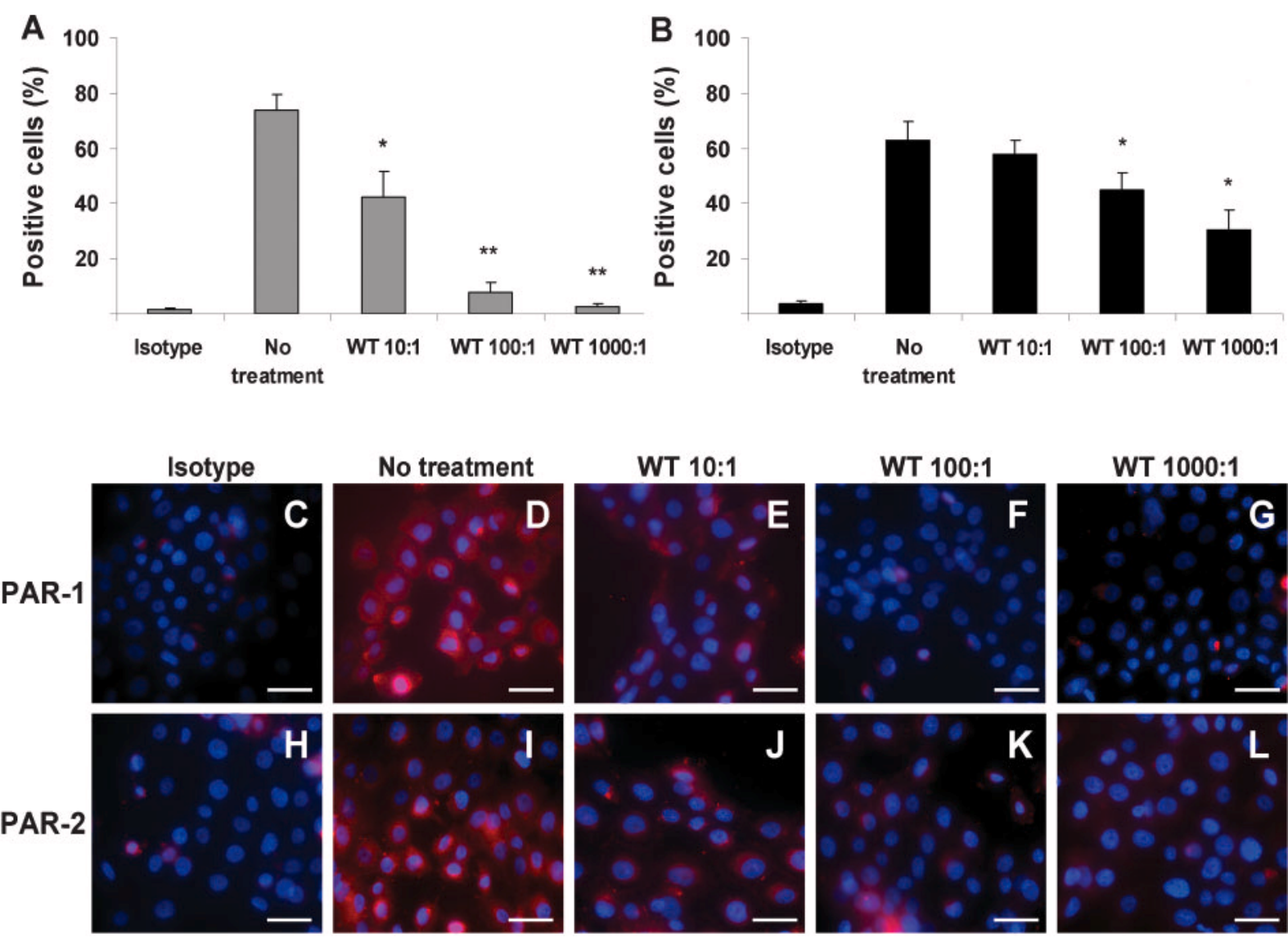

Fig. 3. $P$. gingivalis cleavage of PAR receptors. TERT-2 cells were incubated for $1 \mathrm{~h}$ at $37{ }^{\circ} \mathrm{C}$ in $5 \% \mathrm{CO}_{2}$ with fresh medium or with $P$. gingivalis ATCC 33277 (WT) in spent culture medium at m.o.i. 10:1, $100: 1$ or 1000:1, washed and prepared for flow cytometry $(A, B)$ or immunofluorescence $(C-L)$, as described in Methods. For flow cytometry, cells were detached from the vessels using $0.2 \%(w / v)$ EDTA, fixed, blocked, stained and analysed to determine protease cleavage of PAR-1 (A) and PAR-2 (B) on the cell surface. PAR-1 and PAR-2 cleavage was visualized by immunofluorescence. $P$. gingivalis-exposed (E-G and J$\mathrm{L}$ ) and unexposed (C, D, H, I) cells were fixed, blocked and stained on coverslips for PAR-1 (D-G) or PAR-2 (I-L), as described. Bars represent the mean \pm SEM of three independent experiments. ${ }^{*} P<0.05 ;{ }^{*} P<0.001$, when compared to 'No treatment'. For both assays, $\lg G 1(A, C)$ and $\lg G 2 a(B, H)$ isotype antibodies were used as negative controls. Scale bars, $12 \mu \mathrm{m}$. Blue, DAPI; red, Alexa Fluor 568-conjugated lgG. Fluorescence images are representative of two independent experiments.

cell line, TERT-2, obtained from oral mucosa (Dickson et al., 2000). Immortalized cells behave more consistently in vitro than primary cells from different donors. Unlike HeLa cells, TERT-2 cells retain the phenotype of the original oral keratinocytes. Using indirect approaches, $P$. gingivalis gingipains have been shown to activate PARs. Downstream signalling responses reported include calcium mobilization (Holzhausen et al., 2006; Lourbakos et al., 1998) and cytokine production (Holzhausen et al., 2006; Lourbakos et al., 2001a; Tancharoen et al., 2005). We now provide direct evidence of PAR receptor cleavage by argand lys-gingipains using flow cytometry and immunofluorescence. Binding of specific antibody indicates that the extracellular domain of a PAR is intact and loss of binding shows that the domain has been cleaved. Consistent with previous reports (Lourbakos et al., 2001a), resting, untreated keratinocytes show high expression of PAR-1 and PAR-2 and weak expression of PAR-3 (Fig. 1). PAR-4 has not been described in the oral epithelium (Lourbakos et al., 2001a). After incubation with thrombin, trypsin or $P$. gingivalis cells, ectodomain-specific PAR-1 and -2 antibodies fail to bind, indicating receptor cleavage.

Like the wild-type strain, the gingipain-deletion mutants are assumed to express LPS, although we cannot rule out alternative variants. Since the TLRs are intact and functional on the oral keratinocytes (Giacaman et al., 2007), differences in keratinocyte responses to gingipain mutants are unlikely to be attributable to differential signalling through the TLRs. In our system, $P$. gingivalis cleaves PAR-1 (Fig. 3A and E-G) and PAR-2 (Fig. 3B and $\mathrm{J}-\mathrm{L})$. A $P$. gingivalis mutant strain that expresses neither 

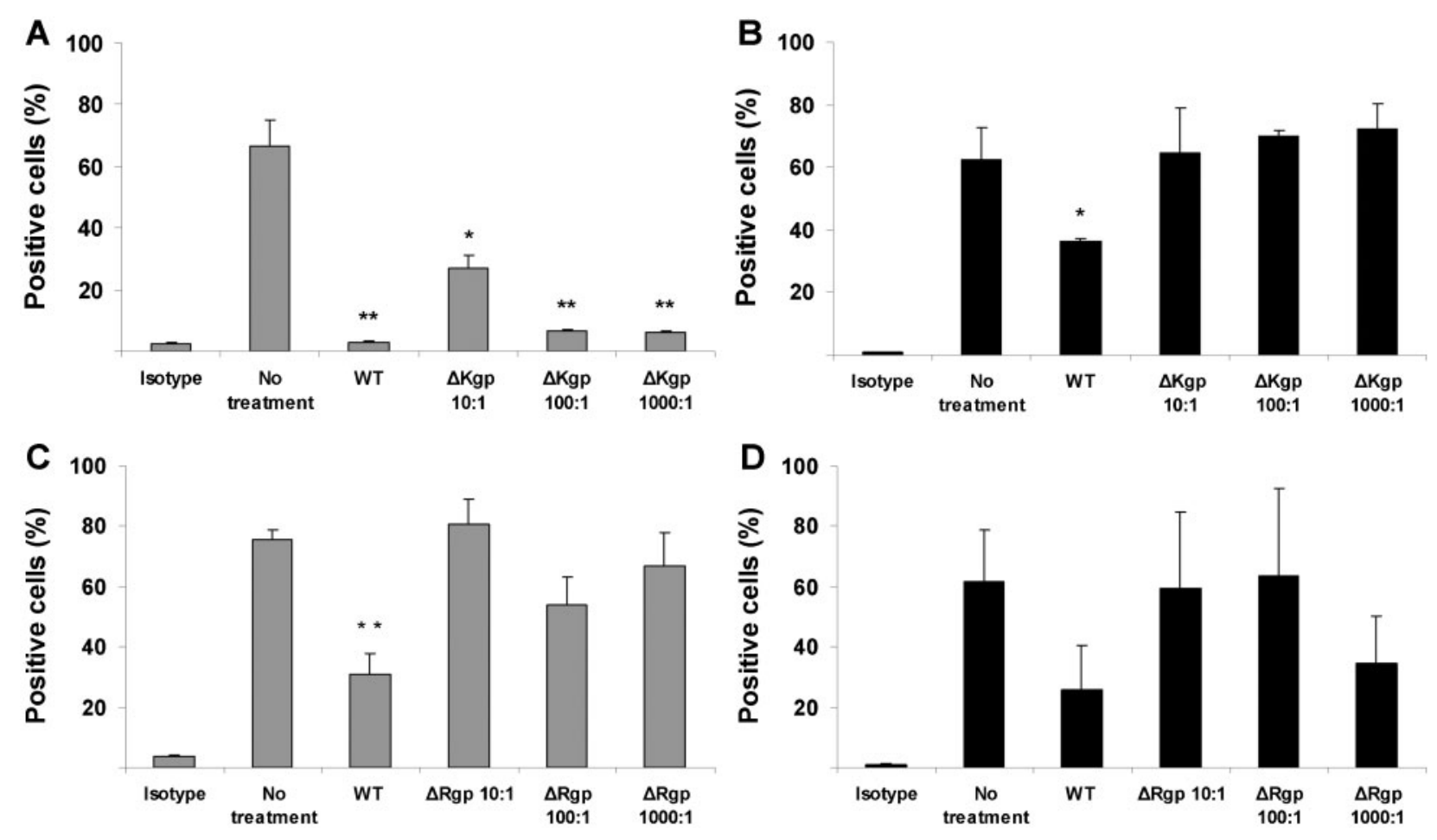

Fig. 4. Arg- and Lys-gingipain specific PAR proteolysis. Oral keratinocytes were incubated for $1 \mathrm{~h}$ with fresh medium (Isotype and No treatment), at m.o.i. $1000: 1$ of $P$. gingivalis ATCC 33277 or at m.o.i. 10:1, $100: 1$ or $1000: 1$ of $P$. gingivalis KDP 129 (A, B) or P. gingivalis KDP 133 (C, D), deletion mutants for Lys- and Arg-gingipains, respectively. Cells were analysed for PAR$1(\mathrm{~A}, \mathrm{C})$ and PAR-2 (B, D) using flow cytometry as described in the legend of Fig. 3 . Wild-type $P$. gingivalis and isotype antibodies, as described in Fig. 3, were used as positive and negative controls, respectively. Graphs depict mean \pm SEM of three independent experiments. ${ }^{\star} P<0.05 ;{ }^{\star \star} P<0.001$, when compared to 'No treatment'.

gingipain fails to cleave PARs on oral keratinocytes, suggesting cleavage by gingipains. Since $P$. gingivalis Rgp and Kgp cannot individually simulate PAR-2 cleavage induced by the wild-type (Fig. 4B, D), the two gingipains might act synergistically to cleave and activate PAR-2. Yet, $P$. gingivalis KDP $129(\Delta \mathrm{Kgp})$ alone cleaves PAR-1 at an m.o.i. similar to $P$. gingivalis ATCC 33277 (Fig. 4A). Rgp is therefore suggested to cleave PAR-1 more effectively than PAR-2. Kgp has also been reported to be abnormally processed in an Rgp mutant (Kadowaki et al., 1998). Furthermore, only Rgp possesses the specificity to cleave the PAR-2-tethered ligand between $\mathrm{Arg}^{34}$ and $\mathrm{Ser}^{35}$ (Uehara et al., 2005). Abnormal processing of Kgp could explain why neither the Rgp mutant nor the double gingipain mutant could simulate the PAR-2 cleavage activity of the wild-type strain. Alternatively, the cleavage of PAR-2 could result from digestion by either of the gingipains acting synergistically or with other $P$. gingivalis proteases. Activation and downstream signalling may depend on either Kgp or Rgp, which may explain the attenuated effect of the mutant strains on PAR-2 cleavage.

How selective cleavage of PAR-1 or -2 contributes to differential downstream signalling may be less clear. Although PAR-2 appears relatively insensitive to cleavage by Rgp (Fig. 4B), Rgp activation and downstream signalling appears to occur through PAR-2 (Holzhausen et al., 2005, 2006; Lourbakos et al., 1998, 2001a; Tancharoen et al., 2005). Hence, the PARs may interact functionally, resulting in pathway cross-talk at some level of signalling, since activation of PAR-1 can co-activate PAR-2 (Ossovskaya \& Bunnett, 2004). In primary endothelial cells, specific PAR-1 antagonists fail to completely suppress PAR-1 activation by thrombin. When PAR-2 is also blocked, however, PAR-1 signalling by thrombin is totally abrogated, suggesting transactivation of PAR-2 by cleaved PAR-1 (O'Brien et al., 2000). Conditions in these experiments were similar to our studies. Thus, functional interactions are likely to occur between PAR-1 and PAR-2 signalling pathways.

The release of pro-inflammatory cytokines by keratinocytes represents an innate immune response to pathogens and their virulence factors. Consistent with previous reports (Lourbakos et al., 2001a; Sandros et al., 2000; Uehara et al., 2008a), we show here that the $P$. gingivalis virulence factor Rgp, but not Kgp, selectively induces the expression of proinflammatory cytokine mRNA by oral epithelial cells. Cytokine regulation was only verified at the mRNA level. Further studies appear necessary to also show regulation at 

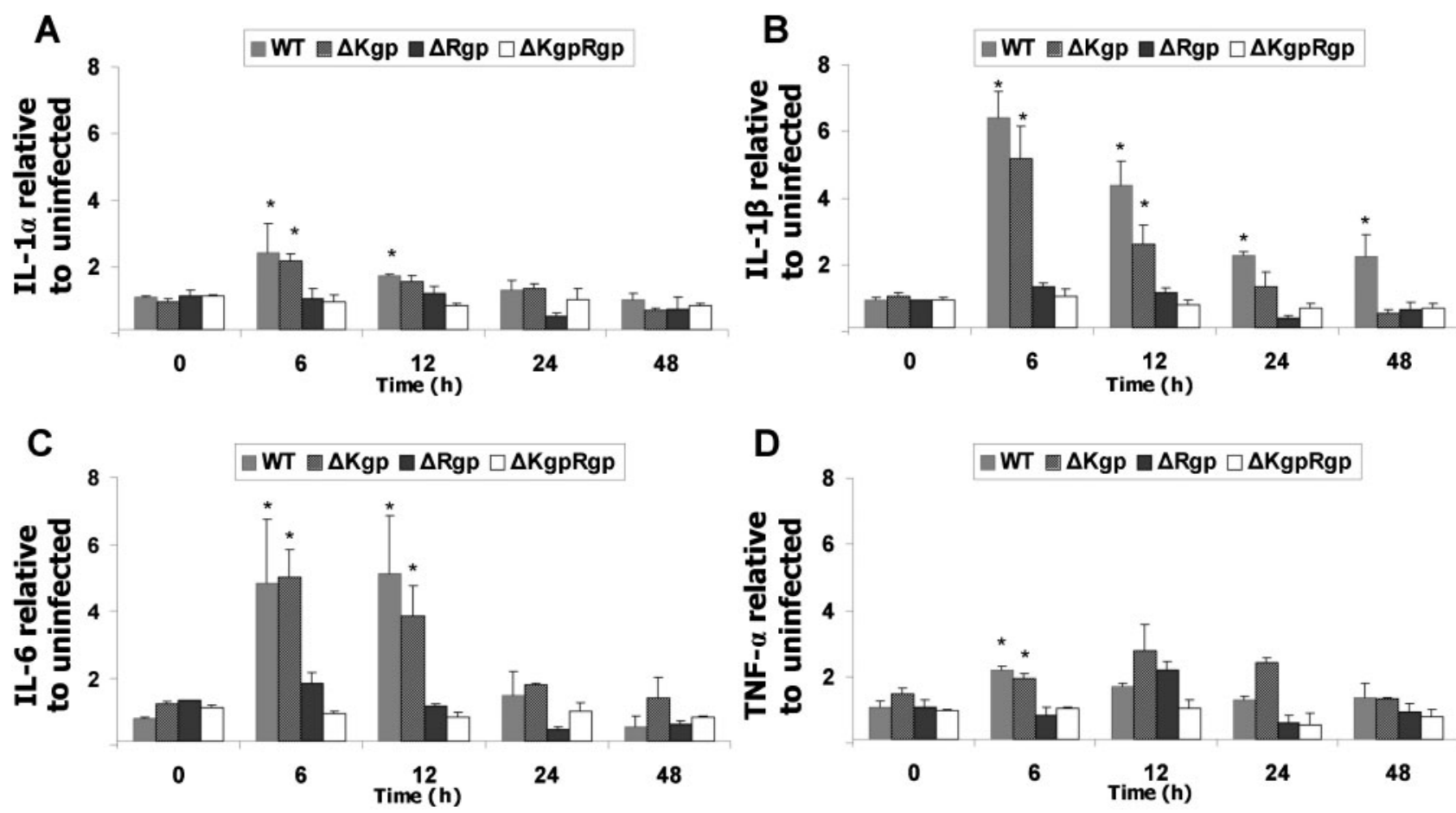

$\square \mathrm{WT} \square \mathrm{aKgp} \square \Delta \mathrm{Rgp} \square \Delta \mathrm{KgpRgp}$

Fig. 5. Induction of pro-inflammatory cytokine mRNAs by $P$. gingivalis and gingipain mutants. TERT-2 cells were incubated at m.o.i. 100:1 with P. gingivalis ATCC 33277, KDP 129, KDP 133 or KDP 136 in spent bacterial culture medium for $3 \mathrm{~h}$, washed and maintained in fresh medium for the indicated total times. At indicated times, cells were harvested and RNA extracted to perform real-time PCR for IL-1 $\alpha$ (A), IL-1 $\beta$ (B), IL-6 (C) and TNF- $\alpha$ (D). $\beta$-Actin was used as a housekeeping gene. Graphs show expression of each molecule relative to uninfected TERT-2 cells harvested at the same indicated time. ${ }^{\star} P<0.05$, when compared to time 0 .

the protein level. Indeed, the gingipains appear to activate PAR-1 to selectively signal the secretion of certain cytokines. Although IL- 6 was specifically induced by Rgp, IL-8 was unaffected by either gingipain. A recent report (Uehara et al., 2008b) using the human oral carcinoma cell line HSC- 2 suggests that IL- 8 is differentially regulated by the purified gingipains. In HSC-2 cells, IL-8 appears to be downregulated by Kgp and HRgpA, while Rgp seems to be upregulated (Uehara et al., 2008b). The immortalized keratinocytes in our study originated from the normal mucosa of the floor of the mouth. Differences in IL-8 responses could be explained by phenotypic differences in the cells but may also reflect differences in the concentrations of specific gingipains associated with freshly harvested $P$. gingivalis and when used as purified proteins in optimized conditions.

The target cell phenotype and co-expressed virulence factors can affect the apparent selectivity of gingipains in upregulating cytokines. For example, $P$. gingivalis Rgp upregulates IL-8 expression in gingival fibroblasts (OidoMori et al., 2001), but not in oral epithelial cells as we show here. Furthermore, $P$. gingivalis gingipains are generally copresented with LPS, which can synergize or simulate PARmediated transcriptional responses in oral keratinocytes
(Giacaman et al., 2007). Since all of the $P$. gingivalis mutants used in our study express LPS, differential regulation of pro-inflammatory cytokines argues in favour of gingipain-associated cytokine induction signalled by selective PAR cleavage, rather than from synergistic LPSmediated activation of TLRs. We cannot, however, rule out that the LPS on the gingipain-mutant strains and the resulting responses in the oral keratinocytes may be altered, given that LPS-gingipain complex formation may mask functional domains of LPS (Takii et al., 2005).

In this complex environment, $P$. gingivalis gingipains have been implicated in the pathogenesis of periodontal disease. $P$. gingivalis-containing dental plaque resides proximal to sulcular gingival keratinocytes, suggesting that activation of PARs and upregulation of pro-inflammatory cytokines could contribute to acute and chronic inflammation in periodontitis. Locally released cytokines can also circulate in the blood (Nagatomo et al., 2007), and P. gingivalis gingipains have been associated with platelet aggregation (Lourbakos et al., 2001b), a key event in thrombosis. In mice, systemic inflammation is promoted by gingipain activation of PAR-2 (Holzhausen et al., 2005, 2006). P. gingivalis invasion and systemic dissemination of gingipains could therefore link periodontal disease and cardiovascular disease. 


\section{ACKNOWLEDGEMENTS}

These studies were supported by NIH/NIDCR grants R01DE11831 and R01DE015503.

\section{REFERENCES}

Chen, T., Nakayama, K., Belliveau, L. \& Duncan, M. J. (2001). Porphyromonas gingivalis gingipains and adhesion to epithelial cells. Infect Immun 69, 3048-3056.

Chen, Y. F., Yan, J., Zhang, D. Y. \& Chen, L. L. (2008). Effect of Porphyromonas gingivalis lipopolysaccharide on induced secretion of inflammatory cytokines by different cell lines. Zhejiang Da Xue Xue Bao Yi Xue Ban 37, 622-628.

Coughlin, S. R. (2000). Thrombin signalling and protease-activated receptors. Nature 407, 258-264.

Dickson, M. A., Hahn, W. C., Ino, Y., Ronfard, V., Wu, J. Y., Weinberg, R. A., Louis, D. N., Li, F. P. \& Rheinwald, J. G. (2000). Human keratinocytes that express hTERT and also bypass a p16(INK4a)-enforced mechanism that limits life span become immortal yet retain normal growth and differentiation characteristics. Mol Cell Biol 20, 1436-1447.

Diya, Z., Lili, C., Shenglai, L., Zhiyuan, G. \& Jie, Y. (2008). Lipopolysaccharide (LPS) of Porphyromonas gingivalis induces ILlbeta, TNF-alpha and IL-6 production by THP-1 cells in a way different from that of Escherichia coli LPS. Innate Immun 14, 99-107.

Eick, S., Reissmann, A., Rodel, J., Schmidt, K. H. \& Pfister, W. (2006). Porphyromonas gingivalis survives within $\mathrm{KB}$ cells and modulates inflammatory response. Oral Microbiol Immunol 21, 231-237.

Eskan, M. A., Rose, B. G., Benakanakere, M. R., Zeng, Q., Fujioka, D., Martin, M. H., Lee, M. J. \& Kinane, D. F. (2008). TLR4 and S1P receptors cooperate to enhance inflammatory cytokine production in human gingival epithelial cells. Eur J Immunol 38, 1138-1147.

Giacaman, R. A., Nobbs, A. H., Ross, K. F. \& Herzberg, M. C. (2007). Porphyromonas gingivalis selectively up-regulates the HIV-1 coreceptor CCR5 in oral keratinocytes. J Immunol 179, 2542-2550.

Hansen, K. K., Saifeddine, M. \& Hollenberg, M. D. (2004). Tethered ligand-derived peptides of proteinase-activated receptor 3 (PAR3) activate PAR1 and PAR2 in Jurkat T cells. Immunology 112, 183-190.

Holzhausen, M., Spolidorio, L. C. \& Vergnolle, N. (2005). Role of protease-activated receptor-2 in inflammation, and its possible implications as a putative mediator of periodontitis. Mem Inst Oswaldo Cruz 100 (Suppl 1), 177-180.

Holzhausen, M., Spolidorio, L. C., Ellen, R. P., Jobin, M. C., Steinhoff, M., Andrade-Gordon, P. \& Vergnolle, N. (2006). Protease-activated receptor-2 activation: a major role in the pathogenesis of Porphyromonas gingivalis infection. Am J Pathol 168, 1189-1199.

Kadowaki, T. \& Yamamoto, K. (2003). Suppression of virulence of Porphyromonas gingivalis by potent inhibitors specific for gingipains. Curr Protein Pept Sci 4, 451-458.

Kadowaki, T., Yoneda, M., Okamoto, K., Maeda, K. \& Yamamoto, K. (1994). Purification and characterization of a novel arginine-specific cysteine proteinase (argingipain) involved in the pathogenesis of periodontal disease from the culture supernatant of Porphyromonas gingivalis. J Biol Chem 269, 21371-21378.

Kadowaki, T., Nakayama, K., Yoshimura, F., Okamoto, K., Abe, N. \& Yamamoto, K. (1998). Arg-gingipain acts as a major processing enzyme for various cell surface proteins in Porphyromonas gingivalis. J Biol Chem 273, 29072-29076.

Kadowaki, T., Takii, R., Baba, A. \& Yamamoto, K. (2003). Gingipains as the determinants of periodontopathogenicity. Nippon Yakurigaku Zasshi 122, 37-44.
Kadowaki, T., Baba, A., Abe, N., Takii, R., Hashimoto, M., Tsukuba, T., Okazaki, S., Suda, Y., Asao, T. \& Yamamoto, K. (2004). Suppression of pathogenicity of Porphyromonas gingivalis by newly developed gingipain inhibitors. Mol Pharmacol 66, 1599-1606.

Livak, K. J. \& Schmittgen, T. D. (2001). Analysis of relative gene expression data using real-time quantitative PCR and the 2(-Delta Delta C(T)) method. Methods 25, 402-408.

Lourbakos, A., Chinni, C., Thompson, P., Potempa, J., Travis, J., Mackie, E. J. \& Pike, R. N. (1998). Cleavage and activation of proteinase-activated receptor-2 on human neutrophils by gingipain- $\mathrm{R}$ from Porphyromonas gingivalis. FEBS Lett 435, 45-48.

Lourbakos, A., Potempa, J., Travis, J., D’Andrea, M. R., AndradeGordon, P., Santulli, R., Mackie, E. J. \& Pike, R. N. (2001a). Argininespecific protease from Porphyromonas gingivalis activates proteaseactivated receptors on human oral epithelial cells and induces interleukin-6 secretion. Infect Immun 69, 5121-5130.

Lourbakos, A., Yuan, Y. P., Jenkins, A. L., Travis, J., Andrade-Gordon, P., Santulli, R., Potempa, J. \& Pike, R. N. (2001b). Activation of proteaseactivated receptors by gingipains from Porphyromonas gingivalis leads to platelet aggregation: a new trait in microbial pathogenicity. Blood 97, 3790-3797.

Madianos, P. N., Papapanou, P. N., Nannmark, U., Dahlen, G. \& Sandros, J. (1996). Porphyromonas gingivalis FDC381 multiplies and persists within human oral epithelial cells in vitro. Infect Immun 64, 660-664.

Miura, M., Hamachi, T., Fujise, O. \& Maeda, K. (2005). The prevalence and pathogenic differences of Porphyromonas gingivalis fimA genotypes in patients with aggressive periodontitis. J Periodontal Res 40, 147-152.

Nagatomo, Y., Yoshikawa, T., Kohno, T., Yoshizawa, A., Anzai, T., Meguro, T., Satoh, T. \& Ogawa, S. (2007). Effects of beta-blocker therapy on high sensitivity c-reactive protein, oxidative stress, and cardiac function in patients with congestive heart failure. J Card Fail 13, 365-371.

Nakayama, K., Kadowaki, T., Okamoto, K. \& Yamamoto, K. (1995). Construction and characterization of arginine-specific cysteine proteinase (Arg-gingipain)-deficient mutants of Porphyromonas gingivalis. Evidence for significant contribution of Arg-gingipain to virulence. J Biol Chem 270, 23619-23626.

Nisapakultorn, K., Ross, K. F. \& Herzberg, M. C. (2001). Calprotectin expression in vitro by oral epithelial cells confers resistance to infection by Porphyromonas gingivalis. Infect Immun 69, 4242-4247.

Nonnenmacher, C., Dalpke, A., Mutters, R. \& Heeg, K. (2004). Quantitative detection of periodontopathogens by real-time PCR. J Microbiol Methods 59, 117-125.

Nonnenmacher, C., Dalpke, A., Rochon, J., Flores-de-Jacoby, L., Mutters, R. \& Heeg, K. (2005). Real-time polymerase chain reaction for detection and quantification of bacteria in periodontal patients. J Periodontol 76, 1542-1549.

O’Brien, P. J., Prevost, N., Molino, M., Hollinger, M. K., Woolkalis, M. J., Woulfe, D. S. \& Brass, L. F. (2000). Thrombin responses in human endothelial cells. Contributions from receptors other than PAR1 include the transactivation of PAR2 by thrombin-cleaved PAR1. J Biol Chem 275, 13502-13509.

Oido-Mori, M., Rezzonico, R., Wang, P. L., Kowashi, Y., Dayer, J. M., Baehni, P. C. \& Chizzolini, C. (2001). Porphyromonas gingivalis gingipain-R enhances interleukin-8 but decreases gamma interferoninducible protein 10 production by human gingival fibroblasts in response to T-cell contact. Infect Immun 69, 4493-4501.

Okamoto, K., Nakayama, K., Kadowaki, T., Abe, N., Ratnayake, D. B. \& Yamamoto, K. (1998). Involvement of a lysine-specific cysteine proteinase in hemoglobin adsorption and heme accumulation by Porphyromonas gingivalis. J Biol Chem 273, 21225-21231. 
Ossovskaya, V. S. \& Bunnett, N. W. (2004). Protease-activated receptors: contribution to physiology and disease. Physiol Rev 84, 579-621.

Pathirana, R. D., O’Brien-Simpson, N. M., Brammar, G. C., Slakeski, N. \& Reynolds, E. C. (2007a). Kgp and RgpB, but not RgpA, are important for Porphyromonas gingivalis virulence in the murine periodontitis model. Infect Immun 75, 1436-1442.

Pathirana, R. D., O'Brien-Simpson, N. M., Visvanathan, K., Hamilton, J. A. \& Reynolds, E. C. (2007b). Flow cytometric analysis of adherence of Porphyromonas gingivalis to oral epithelial cells. Infect Immun 75, 2484-2492.

Potempa, J. \& Pike, R. (2009). Corruption of innate immunity by bacterial proteases. J Innate Immun 1, 70-87.

Potempa, J., Banbula, A. \& Travis, J. (2000). Role of bacterial proteinases in matrix destruction and modulation of host responses. Periodontol 2000 24, 153-192.

Sandros, J., Karlsson, C., Lappin, D. F., Madianos, P. N., Kinane, D. F. \& Papapanou, P. N. (2000). Cytokine responses of oral epithelial cells to Porphyromonas gingivalis infection. J Dent Res 79, 1808-1814.

Shi, Y., Ratnayake, D. B., Okamoto, K., Abe, N., Yamamoto, K. \& Nakayama, K. (1999). Genetic analyses of proteolysis, hemoglobin binding, and hemagglutination of Porphyromonas gingivalis. Construction of mutants with a combination of $\operatorname{rgp} A, \operatorname{rgp} B, \mathrm{kgp}$, and hagA. J Biol Chem 274, 17955-17960.

Takii, R., Kadowaki, T., Baba, A., Tsukuba, T. \& Yamamoto, K. (2005). A functional virulence complex composed of gingipains, adhesins, and lipopolysaccharide shows high affinity to host cells and matrix proteins and escapes recognition by host immune systems. Infect Immun 73, 883-893.

Tancharoen, S., Sarker, K. P., Imamura, T., Biswas, K. K., Matsushita, K., Tatsuyama, S., Travis, J., Potempa, J., Torii, M. \& Maruyama, I. (2005). Neuropeptide release from dental pulp cells by
RgpB via proteinase-activated receptor-2 signaling. J Immunol 174, 5796-5804.

Tatakis, D. N. \& Kumar, P. S. (2005). Etiology and pathogenesis of periodontal diseases. Dent Clin North Am 49, 491-516 (v.).

Uehara, A., Muramoto, K., Imamura, T., Nakayama, K., Potempa, J., Travis, J., Sugawara, S. \& Takada, H. (2005). Arginine-specific gingipains from Porphyromonas gingivalis stimulate production of hepatocyte growth factor (scatter factor) through protease-activated receptors in human gingival fibroblasts in culture. J Immunol 175, 6076-6084.

Uehara, A., Imamura, T., Potempa, J., Travis, J. \& Takada, H. (2008a). Gingipains from Porphyromonas gingivalis synergistically induce the production of proinflammatory cytokines through protease-activated receptors with Toll-like receptor and NOD1/2 ligands in human monocytic cells. Cell Microbiol 10, 1181-1189.

Uehara, A., Naito, M., Imamura, T., Potempa, J., Travis, J., Nakayama, K. \& Takada, H. (2008b). Dual regulation of interleukin-8 production in human oral epithelial cells upon stimulation with gingipains from Porphyromonas gingivalis. J Med Microbiol 57, 500-507.

Wang, P. L., Oido-Mori, M., Fujii, T., Kowashi, Y., Kikuchi, M., Suetsugu, Y., Tanaka, J., Azuma, Y., Shinohara, M. \& Ohura, K. (2002). Effect of anti-CD14 antibody on experimental periodontitis induced by Porphyromonas gingivalis lipopolysaccharide. Jpn J Pharmacol 89, 176-183.

Weinberg, A., Belton, C. M., Park, Y. \& Lamont, R. J. (1997). Role of fimbriae in Porphyromonas gingivalis invasion of gingival epithelial cells. Infect Immun 65, 313-316.

Yoneda, M., Maeda, K. \& Aono, M. (1990). Suppression of bactericidal activity of human polymorphonuclear leukocytes by Bacteroides gingivalis. Infect Immun 58, 406-411.

Edited by: P. Kolenbrander 